\title{
Large-scale Cloze Test Dataset Created by Teachers
}

\author{
Qizhe Xie*, Guokun Lai*, Zihang Dai, Eduard Hovy \\ Language Technologies Institute, Carnegie Melon University \\ \{qizhex, guokun, dzihang, hovy\}@cs.cmu.edu
}

\begin{abstract}
Cloze tests are widely adopted in language exams to evaluate students' language proficiency. In this paper, we propose the first large-scale human-created cloze test dataset CLOTH ${ }^{1}{ }^{2}$, containing questions used in middle-school and high-school language exams. With missing blanks carefully created by teachers and candidate choices purposely designed to be nuanced, CLOTH requires a deeper language understanding and a wider attention span than previously automaticallygenerated cloze datasets. We test the performance of dedicatedly designed baseline models including a language model trained on the One Billion Word Corpus and show humans outperform them by a significant margin. We investigate the source of the performance gap, trace model deficiencies to some distinct properties of CLOTH, and identify the limited ability of comprehending the long-term context to be the key bottleneck.
\end{abstract}

\section{Introduction}

Being a classic language exercise, the cloze test (Taylor, 1953) is an accurate assessment of language proficiency (Fotos, 1991; Jonz, 1991; Tremblay, 2011) and has been widely employed in language examinations. Under a typical setting, a cloze test requires examinees to fill in missing words (or sentences) to best fit the surrounding context. To facilitate natural language understanding, automatically-generated cloze datasets are introduced to measure the ability of machines in reading comprehension (Hermann et al., 2015; Hill et al., 2016; Onishi et al., 2016). In these datasets, each cloze question typically consists of

\footnotetext{
${ }^{*}$ Equal contribution.

${ }^{1} \mathrm{CLOTH}$ (CLOze test by TeacHers) is available at http://www.cs.cmu.edu/ glail/data/cloth/.

${ }^{2}$ The leaderboard is available at http://www. qizhexie.com/data/CLOTH_leaderboard.html
}

a context paragraph and a question sentence. By randomly replacing a particular word in the question sentence with a blank symbol, a single test case is created. For instance, CNN/Daily Mail datasets (Hermann et al., 2015) use news articles as contexts and summary bullet points as the question sentence. Only named entities are removed when creating the blanks. Similarly, in Children's Books test (CBT) (Hill et al., 2016), cloze questions are obtained by removing a word in the last sentence of every consecutive 21 sentences, with the first 20 sentences being the context. Different from CNN/Daily Mail datasets, CBT also provides each question with a candidate answer set, consisting of randomly sampled words with the same part-of-speech tag from the context as that of the correct answer.

Thanks to the automatic generation process, these datasets can be very large in size, leading to significant research progresses. However, compared to how humans would create cloze questions and evaluate reading comprehension ability, the automatic generation process bears some inevitable issues. Firstly, blanks are chosen uniformly without considering which aspect of the language phenomenon that questions will test. Hence, quite a portion of automatically-generated questions can be purposeless or even trivial to answer. Another issue involves the ambiguity of answers. Given a context and a sentence with a blank, there can be multiple words that fit almost equally well into the blank. A possible solution is to include a candidate option set, as done by CBT, to get rid of the ambiguity. However, automatically generating the candidate option set can be problematic since it cannot guarantee the ambiguity is removed. More importantly, automaticallygenerated candidates can be totally irrelevant or simply grammatically unsuitable for the blank, resulting in again purposeless or trivial questions. 
Probably due to these unsatisfactory issues, neural models have achieved comparable results to the human-level performance within a very short time (Chen et al., 2016; Dhingra et al., 2016; Seo et al., 2016). While there have been works trying to incorporate human design into cloze question generation (Zweig and Burges, 2011; Paperno et al., 2016), due to the expensive labeling process, the MSR Sentence Completion Challenge created by this effort has 1,040 questions and the LAMBADA (Paperno et al., 2016) dataset has 10,022 questions, limiting the possibility of developing powerful neural models on it. As a result of the small size, human-created questions are only used to compose development sets and test sets. Motivated by the aforementioned drawbacks, we propose CLOTH, a large-scale cloze test dataset collected from English exams. Questions in the dataset are designed by middle-school and highschool teachers to prepare Chinese students for entrance exams. To design a cloze test, teachers firstly determine the words that can test students' knowledge of vocabulary, reasoning or grammar; then replace those words with blanks and provide other three candidate options for each blank. If a question does not specifically test grammar usage, all of the candidate options would complete the sentence with correct grammar, leading to highly nuanced questions. As a result, human-created questions are usually harder and are a better assessment of language proficiency. A general cloze test evaluates several aspects of language proficiency including vocabulary, reasoning and grammar, which are key components of comprehending natural language.

To verify if human-created cloze questions are difficult for current models, we train and evaluate the state-of-the-art language model (LM) and machine comprehension models on this dataset, including a language model trained on the One Billion Word Corpus. We find that the state-of-theart model lags behind human performance even if the model is trained on a large external corpus. We analyze where the model fails compared to humans who perform well. After conducting error analysis, we assume the performance gap results from the model's inability to use a long-term context. To examine this assumption, we evaluate human-level performance when the human subjects are only allowed to see one sentence as the context. Our assumption is confirmed by the matched performances of the models and human when given only one sentence. In addition, we demonstrate that human-created data is more difficult than automatically-generated data. Specifically, it is much easier for the same model to perform well on automatically-generated data.

We hope that CLOTH provides a valuable testbed for both the language modeling community and the machine comprehension community. Specifically, the language modeling community can use CLOTH to evaluate their models' abilities in modeling long contexts, while the machine comprehension community can use CLOTH to test machine's understanding of language phenomena.

\section{Related Work}

Large-scale automatically-generated cloze tests (Hermann et al., 2015; Hill et al., 2016; Onishi et al., 2016) lead to significant research advancements. However, generated questions do not consider language phenomenon to be tested and are relatively easy to solve. Recently proposed reading comprehension datasets are all labeled by humans to ensure a high quality (Rajpurkar et al., 2016; Joshi et al., 2017; Trischler et al., 2016; Nguyen et al., 2016).

Perhaps the closet work to CLOTH is the LAMBADA dataset (Paperno et al., 2016). LAMBADA also targets at finding challenging words to test LM's ability in comprehending a longer context. However, LAMBADA does not provide a candidate set for each question, which can cause ambiguities when multiple words can fit in. Furthermore, only test set and development set are labeled manually. The provided training set is the unlabeled Book Corpus (Zhu et al., 2015). Such unlabeled data do not emphasize long-dependency questions and have a mismatched distribution with the test set, as showed in Section 5. Further, the Book Corpus is too large to allow rapid algorithm development for researchers who do not have access to a huge amount of computational power.

Aiming to evaluate machines under the same conditions that the humans are evaluated, there is a growing interest in obtaining data from examinations. NTCIR QA Lab (Shibuki et al., 2014) contains a set of real-world college entrance exam questions. The Entrance Exams task at CLEF QA Track (Peñas et al., 2014; Rodrigo et al., 2015) evaluates machine's reading comprehension abil- 
ity. The AI2 Reasoning Challenge (Clark et al., 2018; Schoenick et al., 2017) contains approximately eight thousand scientific questions used in middle school. Lai et al. (2017) proposes the first large-scale machine comprehension dataset obtained from exams. They show that questions designed by teachers have a significantly larger proportion of reasoning questions. Our dataset focuses on evaluating both language proficiency and reasoning abilities.

\section{CLOTH Dataset}

In this section, we introduce the CLOTH dataset that is collected from English examinations, and study its abilities of assessment.

\subsection{Data Collection and Statistics}

We collect the raw data from three free and public websites in China that gather exams created by English teachers to prepare students for college/high school entrance exams ${ }^{3}$. Before cleaning, there are 20,605 passages and 332, 755 questions. We perform the following processes to ensure the validity of data: Firstly, we remove questions with an inconsistent format such as questions with more than four options. Then we filter all questions whose validity relies on external information such as pictures or tables. Further, we find that half of the total passages are duplicates and we delete those passages. Lastly, on one of the websites, the answers are stored as images. We use two OCR software programs ${ }^{4}$ to extract the answers from images. We discard the questions when results from the two software are different. After the cleaning process, we obtain a clean dataset of 7, 131 passages and 99, 433 questions.

Since high school questions are more difficult than middle school questions, we divide the datasets into CLOTH-M and CLOTH-H, which stand for the middle school part and the high school part. We split $11 \%$ of the data for both the test set and the development set. The detailed statistics of the whole dataset and two subsets are presented in Table 1. Note that the questions were created to test non-native speakers, hence the vocabulary size is not very large.

\footnotetext{
3 The three websites include http://www.21cnjy.com/; http://5utk.ks5u.com/; http://zujuan.xkw.com/. We checked that CLOTH does not contain sentence completion example questions from GRE, SAT and PSAT.

${ }^{4}$ tesseract: https://github.com/tesseract-ocr; ABBYY FineReader: https://www.abbyy.com/en-us/finereader/
}

\subsection{Question Type Analysis}

In order to evaluate students' mastery of a language, teachers usually design tests in a way that questions cover different aspects of a language. Specifically, they first identify words in the passage that can examine students' knowledge in vocabulary, logic, or grammar. Then, they replace the words with blanks and prepare three incorrect but nuanced candidate options to make the test non-trivial. A sample passage is presented in Table 2 .

To understand the abilities of assessment on this dataset, we divide questions into several types and label the proportion of each type. According to English teachers who regularly create cloze test questions for English exams in China, there are largely three types: grammar, vocabulary and reasoning. Grammar questions are easily differentiated from other two categories. However, the teachers themselves cannot specify a clear distinction between reasoning questions and vocabulary questions since all questions require comprehending the words within the context and conducting some level of reasoning by recognizing incomplete information or conceptual overlap.

Hence, we divided the questions except grammar questions based on the difficulty level for a machine to answer the question, following works on analyzing machine comprehension datasets (Chen et al., 2016; Trischler et al., 2016). In particular, we divide them in terms of their dependency ranges, since questions that only involve a single sentence are easier to answer than questions involving evidence distributed in multiple sentences. Further, we divided questions involving long-term dependency into matching/paraphrasing questions and reasoning questions since matching questions are easier. The four types include:

- Grammar: The question is about grammar usage, involving tense, preposition usage, active/passive voices, subjunctive mood and so on.

- Short-term-reasoning: The question is about content words and can be answered based on the information within the same sentence. Note that the content words can evaluate knowledge of both vocabulary and reasoning.

- Matching/paraphrasing: The question is answered by copying/paraphrasing a word in the context. 


\begin{tabular}{l|ccc|ccc|ccc}
\hline \multirow{2}{*}{ Dataset } & \multicolumn{3}{|c|}{ CLOTH-M } & \multicolumn{3}{c|}{ CLOTH-H } & \multicolumn{3}{c}{ CLOTH (Total) } \\
& Train & Dev & Test & Train & Dev & Test & Train & Dev & Test \\
\hline \# passages & 2,341 & 355 & 335 & 3,172 & 450 & 478 & 5,513 & 805 & 813 \\
\# questions & 22,056 & 3,273 & 3,198 & 54,794 & 7,794 & 8,318 & 76,850 & 11,067 & 11,516 \\
Vocab. size & & 15,096 & & & 32,212 & & & 37,235 \\
\hline Avg. \# sentence & \multicolumn{3}{c|}{16.26} & & & 18.92 & & \multicolumn{3}{c}{17.79} \\
Avg. \# words & \multicolumn{3}{c}{242.88} & & 365.1 & & \multicolumn{3}{c}{313.16} \\
\hline
\end{tabular}

Table 1: The statistics of the training, development and test sets of CLOTH-M (middle school questions), CLOTH-H (high school questions) and CLOTH

- Long-term-reasoning: The answer must be inferred from synthesizing information distributed across multiple sentences.

We sample 100 passages in the high school category and the middle school category respectively with totally 3,000 questions. The types of these questions are labeled on Amazon Turk. We pay $\$ 1$ and $\$ 0.5$ for high school passages and middle school passages respectively. We refer readers to Appendix A.1 for details of the labeling processes and the labeled sample passage.

The proportion of different questions is shown in Table 3. The majority of questions are shortterm-reasoning questions while approximately $22.4 \%$ of the data needs long-term information, in which the long-term-reasoning questions constitute a large proportion.

\section{Exploring Models' Limits}

In this section, we investigate if human-created cloze test is a challenging problem for state-ofthe-art models. We find that LM trained on the One Billion Word Corpus can achieve a remarkable score but cannot solve the cloze test. After conducting an error analysis, we hypothesize that the model is not able to deal with long-term dependencies. We verify the hypothesis by comparing the model's performance with the human performance when the information humans obtain is limited to one sentence.

\subsection{Human and Model Performance}

LSTM To test the performance of RNN-based supervised models, we train a bidirectional LSTM (Hochreiter and Schmidhuber, 1997) to predict the missing word given the context with only labeled data. The implementation details are in Appendix A.3.

Attentive Readers To enable the model to gather information from a longer context, we aug- ment the supervised LSTM model with the attention mechanism (Bahdanau et al., 2014), so that the representation at the blank is used as a query to find the relevant context in the document and a blank-specific representation of the document is used to score each candidate answer. Specifically, we adapt the Stanford Attentive Reader (Chen et al., 2016) and the positionaware attention model (Zhang et al., 2017) to the cloze test problem. With the position-aware attention model, the attention scores are based on both the context match and the distance from a context to the blank. Both attention models are trained only with human-created blanks just as the LSTM model.

LM In cloze test, the context on both sides may be enough to determine the correct answer. Suppose $x_{i}$ is the missing word and $x_{1}, \cdots, x_{i-1}$, $x_{i+1}, \cdots, x_{n}$ are the context, we choose $x_{i}$ that maximizes the joint probability $p\left(x_{1}, \cdots, x_{n}\right)$, which essentially maximizes the conditional likelihood $p\left(x_{i} \mid x_{1}, \cdots, x_{i-1}, x_{i+1}, \cdots, x_{n}\right)$. Therefore, LM can be naturally adapted to cloze test.

In essence, LM treats each word as a possible blank and learns to predict it. As a result, it receives more supervision than the LSTM trained on human-labeled questions. Besides training a neural LM on our dataset, interested in whether the state-of-the-art LM can solve cloze test, we also test the LM trained on the One Billion Word Benchmark (Chelba et al., 2013) (referred as 1BLM) that achieves a perplexity of 30.0 (Jozefowicz et al., 2016) ${ }^{5}$. To make the evaluation time tractable, we limit the context length to one sentence or three sentences. Note that the One Billion Word Corpus does not overlap with the CLOTH

\footnotetext{
${ }^{5}$ The pre-trained model is obtained from https://github.com/tensorflow/models/tree/master/research/ $1 \mathrm{~m} \_1 \mathrm{~b}$
} 
Passage: Nancy had just got a job as a secretary in a company. Monday was the first day she went to work, so she was very $1_{-}$and arrived early. She $2_{-}$the door open and found nobody there. "I am the $3_{-}$to arrive." She thought and came to her desk. She was surprised to find a bunch of _4_ on it. They were fresh. She $5_{-}$them and they were sweet. She looked around for a $6_{-}$to put them in. "Somebody has sent me flowers the very first day!" she thought $7_{-}$. " But who could it be?" she began to $\_8_{-}$. The day passed quickly and Nancy did everything with _9_ interest. For the following days of the ${ }_{-} 10_{-}$, the first thing Nancy did was to change water for the followers and then set about her work.

Then came another Monday. _ 11 _ she came near her desk she was overjoyed to see a(n) _ $12_{-}$bunch of flowers there. She quickly put them in the vase, _13_ the old ones. The same thing happened again the next Monday. Nancy began to think of ways to find out the _14_. . On Tuesday afternoon, she was sent to hand in a plan to the $1_{-}$. She waited for his directives at his secretary's _ $16_{-}$. She happened to see on the desk a half-opened notebook, which _17_: "In order to keep the secretaries in high spirits, the company has decided that every Monday morning a bunch of fresh flowers should be put on each secretarys desk." Later, she was told that their general manager was a business management psychologist.

\section{Questions:}

1. A. depressed B. encouraged C. excited D. surprised

$\begin{array}{lll}\text { 2. A. turned } & \text { B. pushed } & \text { C. knocked D. forced }\end{array}$

$\begin{array}{llll}\text { 3. A. last } & \text { B. second } & \text { C. third } & \text { D. first }\end{array}$

$\begin{array}{llll}\text { 4. A. keys } & \text { B. grapes } & \text { C. flowers } & \text { D. bananas }\end{array}$

5. A. smelled B. ate C. took D. held

$\begin{array}{llll}\text { 6. A. vase } & \text { B. room } & \text { C. glass } & \text { D. bottle }\end{array}$

$\begin{array}{llll}\text { 7. A. angrily } & \text { B. quietly } & \text { C. strangely } & \text { D. happily }\end{array}$

8. A. seek

9. A. low

B. wonder

B. little

10. A. month

11. A. Unless

12. A. old

B. period

B. When

B. red

C. work

C. great

D. ask

C. year

C. Since

D. general

C. blue

D. week

D. Before

13. A. covering B. demanding C. replacing D. forbidding

14. A. sender B. receiver

15. A. assistant B. colleague

16. A. notebook B. desk

17. A. said

B. written

$\begin{array}{ll}\text { C. secretary D. waiter } & \text { D. }\end{array}$

C. employee D. manager

C. office D. house

C. printed D. signed

Table 2: A Sample passage from our dataset. Bold faces highlight the correct answers. There is only one best answer among four candidates, although several candidates may seem correct.

corpus.

Human performance We measure the performance of Amazon Mechanical Turkers on 3,000 sampled questions when the whole passage is given.

Results The comparison is shown in Table 4. Both attentive readers achieve similar accuracy to the LSTM. We hypothesize that the reason of the attention model's unsatisfactory performance is that the evidence of a question cannot be simply found by matching the context. Similarly, on reading comprehension, though attention-based models (Wang et al., 2017; Seo et al., 2016; Dhingra

\begin{tabular}{l|cc|cc|c}
\hline & \multicolumn{2}{|c|}{ Short-term } & \multicolumn{2}{|c|}{ Long-term } & \\
\hline Dataset & GM & STR & MP & LTR & O \\
\hline CLOTH & 0.265 & 0.503 & 0.044 & 0.180 & 0.007 \\
CLOTH-M & 0.330 & 0.413 & 0.068 & 0.174 & 0.014 \\
CLOTH-H & 0.240 & 0.539 & 0.035 & 0.183 & 0.004 \\
\hline
\end{tabular}

Table 3: The question type statistics of 3000 sampled questions where GM, STR, MP, LTR and O denotes grammar, short-term-reasoning, matching/paraphrasing, long-term-reasoning and others respectively.

\begin{tabular}{l|ccc}
\hline Model & CLOTH & CLOTH-M & CLOTH-H \\
\hline LSTM & 0.484 & 0.518 & 0.471 \\
Stanford AR & 0.487 & 0.529 & 0.471 \\
Position-aware AR & 0.485 & 0.523 & 0.471 \\
\hline LM & 0.548 & 0.646 & 0.506 \\
1B-LM (one sent.) & 0.695 & 0.723 & 0.685 \\
1B-LM (three sent.) & 0.707 & 0.745 & 0.693 \\
\hline Human performance & 0.859 & 0.897 & 0.845 \\
\hline
\end{tabular}

Table 4: Models' performance and human-level performance on CLOTH. LSTM, Stanford Attentive Reader and Attentive Reader with positionaware attention shown in the top part only use supervised data labelled by human. LM outperforms LSTM since it receives more supervisions in learning to predict each word. Training on large external corpus further significantly enhances LM's accuracy.

et al., 2016) have reached human performance on the SQuAD dataset (Rajpurkar et al., 2016), their performance is still not comparable to human performance on datasets that focus more on reasoning where the evidence cannot be simply found by a matching behavior (Lai et al., 2017; Xu et al., 2017). Since the focus of this paper is to analyze the proposed dataset, we leave the design of reasoning oriented attention models for future work.

The LM achieves much better performance than LSTM. The gap is larger when the LM is trained on the 1 Billion Word Corpus, indicating that more training data results in a better generalization. Specifically, the accuracy of 1B-LM is 0.695 when one sentence is used as the context. It indicates that LM can learn sophisticated language regularities when given sufficient data. The same conclusion can also be drawn from the success of a concurrent work ELMo which uses LM representations as word vectors and achieves state-ofthe-art results on six language tasks (Peters et al., 
2018). However, if we increase the context length to three sentences, the accuracy of 1B-LM only has a marginal improvement. In contrast, humans outperform 1B-LM by a significant margin, which demonstrates that deliberately designed questions in CLOTH are not completely solved even for state-of-the-art models.

\subsection{Analyzing 1B-LM's Strengths and Weaknesses}

In this section, we would like to understand why 1B-LM lags behind human performance. We find that most of the errors involve long-term reasoning. Additionally, in a lot of cases, the dependency is within the context of three sentences. We show several errors made by the 1B-LM in Table 5. In the first example, the model does not know that Nancy found nobody in the company means that Nancy was the first one to arrive at the company. In the second and third example, the model fails probably because of not recognizing "they" referred to "flowers". The dependency in the last case is longer. It depends on the fact that Nancy was alone in the company.

Based on the case study, we hypothesize that the LM is not able to take long-term information into account, although it achieves a surprisingly good overall performance. Additionally, the 1BLM is trained on the sentence level, which might also result in the inability to track paragraph level information. However, to investigate the differences between training on sentence level and on paragraph level, a prohibitive amount of computational resource is required to train a large model on the 1 Billion Word Corpus.

On the other hand, a practical comparison is to test the model's performance on different types of questions. We find that the model's accuracy is 0.591 on long-term-reasoning questions of CLOTH-H while it achieves 0.693 on short-termreasoning (a comprehensive type-specific performance is available in Appendix A.3), which partially confirms that long-term-reasoning is harder. However, we could not completely rely on the performance on specific questions types, partly due to a large variance caused by the small sample size. Another reason is that the reliability of question type labels depends on whether turkers are careful enough. For example, in the error analysis shown in Table 5, a careless turker would label the second example as short-term-reasoning without noticing that the meaning of "they" relies on a long context.

To objectively verify if the LM's strengths lie in dealing with short-term information, we obtain the ceiling performance of only utilizing shortterm information. Showing only one sentence as the context, we ask the Turkers to select an option based on their best guesses given the insufficient information. By limiting the context span manually, the ceiling performance with the access to only a short context is estimated accurately.

As shown in Table 6, The performance of 1BLM using one sentence as the context can almost match the human ceiling performance of only using short-term information. Hence we conclude that the LM can almost perfectly solve all shortterm cloze questions. However, the performance of LM is not improved significantly when a longterm context is given, indicating that the performance gap is due to the inability of long-term reasoning.

\section{Comparing Human-created Data and Automatically-generated Data}

In this section, we demonstrate that humancreated data is a better testbed than automaticallygenerated cloze test since it results in a larger gap between model's performance and human performance.

A casual observation is that a cloze test can be created by randomly deleting words and randomly sampling candidate options. In fact, to generate large-scale data, similar generation processes have been introduced and widely used in machine comprehension (Hermann et al., 2015; Hill et al., 2016; Onishi et al., 2016). However, research on cloze test design (Sachs et al., 1997) shows that tests created by deliberately deleting words are more reliable than tests created by randomly or periodically deleting words. To design accurate language proficiency assessment, teachers usually deliberately select words in order to examine students' proficiency in grammar, vocabulary and reasoning. Moreover, in order to make the question non-trivial, three incorrect options provided by teachers are usually grammatically correct and relevant to the context. For instance, in the fourth problem of the sample passage shown in Table 2, "grapes", "flowers" and "bananas" all fit the description of being fresh.

Hence we naturally hypothesize that humangenerated data has distinct characteristics when 


\begin{tabular}{l|llll}
\hline Context & & Options \\
\hline $\begin{array}{l}\text { She pushed the door open and found nobody there. "I am the _- to arrive." She } \\
\text { thought and came to her desk. }\end{array}$ & A. last & B. second & C. third & D. first \\
\hline $\begin{array}{l}\text { They were fresh. She _- them and they were sweet. She looked around for a vase } \\
\text { to put them in. }\end{array}$ & A. smelled & B. ate & C. took & D. held \\
\hline $\begin{array}{l}\text { She smelled them and they were sweet. She looked around for a _- to put them in. } \\
\text { "Somebody has sent me flowers the very first day!" }\end{array}$ & A. vase & B. room & C. glass & D. bottle \\
$\begin{array}{l}\text { "But who could it be?" she began to _- . The day passed quickly and Nancy did } \\
\text { everything with great interest. }\end{array}$ & A. seek & B. wonder & C. work & D. ask \\
\hline
\end{tabular}

Table 5: Error analysis of 1-billion-language-model with three sentences as the context. The questions are sampled from the sample passage shown in Table 2. The correct answer is in bold text. The incorrectly selected options are in italics.

\begin{tabular}{c|l|ccc}
\hline & Model & \multicolumn{3}{|c}{ CLOTH CLOTH-M CLOTH-H } \\
\hline \multirow{2}{*}{ Short context } & 1B-LM & 0.695 & 0.723 & 0.685 \\
& Human & 0.713 & 0.771 & 0.691 \\
\hline \multirow{2}{*}{ Long context } & 1B-LM & 0.707 & 0.745 & 0.693 \\
& Human & 0.859 & 0.897 & 0.845 \\
\hline
\end{tabular}

Table 6: Humans' performance compared with 1billion-language-model. In the short context part, both 1B-LM and humans only use information of one sentence. In the long context part, humans have the whole passage as the context, while 1BLM uses contexts of three sentences.

compared with automatically-generated data. To verify this assumption, we compare the LSTM model's performance when given different proportions of the two types of data. Specifically, to train a model with $\alpha$ percent of automatically-generated data, we randomly replace $a$ percent blanks with blanks at random positions, while keeping the remaining $1-\alpha$ percent questions the same. The candidate options for the generated blanks are random words sampled from the unigram distribution. We test models obtained with varying $\alpha$ on human-created data and automatically-generated data respectively.

\begin{tabular}{|c|c|c|c|c|c|}
\hline${ }_{\text {Test }} \quad \alpha \%$ & $0 \%$ & $25 \%$ & $50 \%$ & $75 \%$ & $100 \%$ \\
\hline human-created & 0.484 & 0.475 & 0.469 & 0.423 & 0.381 \\
\hline Generated & 0.422 & 0.699 & 0.757 & 0.785 & 0.815 \\
\hline
\end{tabular}

Table 7: The model's performance when trained on $\alpha$ percent of automatically-generated data and $100-\alpha$ percent of human-created data

From the comparison in Table 7, we have the following observations: (1) human-created data leads to a larger gap between model's performance and the ceiling/human performance. The model's performance and human's performance on the human-created data are 0.484 and 0.859 respectively, as shown in Tab. 4, leading to a gap of 0.376. In comparison, the performance gap on the automatically-generated data is at most 0.185 since the model's performance reaches an accuracy of 0.815 when fully trained on generated data. (2) Although human-created data may provide more information in distinguishing similar words, the distributional mismatch between two types of data makes it non-trivial to transfer the knowledge gained from human-created data to tackle automatically-generated data. Specifically, the model's performance on automatically-generated data monotonically decreases when given a higher ratio of human-created data.

\section{Combining Human-created Data with Automatically-generated Data}

In Section 4.1, we show that LM is able to take advantage of more supervision since it predicts each word based on the context. At the same time, we also show that human-created data and the automatically-generated data are quite different in Section 5. In this section, we propose a model that takes advantage of both sources.

\subsection{Representative-based Model}

Specifically, for each question, regardless of being human-created or automatically-generated, we can compute the negative log likelihood of the correct answer as the loss function. Suppose $J_{\mathrm{H}}$ is the average negative log likelihood loss for human-created questions and $J_{\mathrm{R}}$ is the loss function on generated questions, we combine losses on human-created questions and generated questions by simply adding them together, i.e., $J_{\mathrm{R}}+J_{\mathrm{H}}$ is used as the final loss function. We will introduce the definition of $J_{\mathrm{R}}$ in the following paragraphs.

Although automatically-generated data has a 
large quantity and is valuable to the model training, as shown in the previous Section, automatically-generated questions are quite different from human-created questions. Ideally, a large amount of human-created questions is more desirable than a large amount of automaticallygenerated questions. A possible avenue towards having large-scale human-created data is to automatically pick out a large number of generated questions which are representative of or similar to human-created questions. In other words, we train a network to predict whether a question is a generated question or a human-created question. A generated question is representative of human-created questions if it has a high probability of being a human-created question. Then we can give higher weights to questions that resemble human-created question.

We first introduce our method to obtain the representativeness information. Let $x$ denote the passage and $z$ denote whether a word is selected as a question by human, i.e., $z$ is 1 if this word is selected to be filled in the original passage or 0 otherwise. Suppose $h_{i}$ is the representation of $i$-th word given by a bidirectional LSTM. The network computes the probability $p_{i}$ of $x_{i}$ being a humancreated question as follows:

$$
l_{i}=h_{i}^{T} w_{x_{i}} ; \quad p_{i}=\operatorname{Sigmoid}\left(l_{i}\right)
$$

where $l_{i}$ is the logit which will be used as in the final model and $w_{x_{i}}$ is the the word embedding. We train the network to minimize the binary cross entropy between $p$ and ground-truth labels at each token.

After obtaining the representativeness information, we define the representativeness weighted loss function as

$$
J_{\mathrm{R}}=\sum_{i \notin H} \operatorname{Softmax}_{i}\left(\frac{l_{1}}{\alpha}, \cdots, \frac{l_{n}}{\alpha}\right) J_{i}
$$

where $J_{i}$ denotes the negative log likelihood loss for the $i$-th question and let $l_{i}$ be the output representativeness of the $i$-th question and $H$ is the set of all human-generated questions and $\alpha$ is the temperature of the Softmax function. The model degenerates into assigning a uniform weight to all questions when the temperature is $+\infty$. We set $\alpha$ to 2 based on the performance on the dev set. ${ }^{6}$.

\footnotetext{
${ }^{6}$ The code is available at https://github.com/qizhex/Largescale-Cloze-Test-Dataset-Created-by-Teachers
}

\begin{tabular}{l|c|ccc}
\hline Model & Ex. & CLOTH & CLOTH-M & CLOTH-H \\
\hline Our model & & $\mathbf{0 . 5 8 3}$ & $\mathbf{0 . 6 7 3}$ & $\mathbf{0 . 5 4 9}$ \\
LM & \multirow{2}{*}{ No } & 0.548 & 0.646 & 0.506 \\
LSTM & 0.484 & 0.518 & 0.471 \\
Stanford AR & & 0.487 & 0.529 & 0.471 \\
\hline 1B-LM & Yes & 0.707 & 0.745 & 0.693 \\
\hline Human & & 0.859 & 0.897 & 0.845 \\
\hline
\end{tabular}

Table 8: Overall results on CLOTH. Ex. denotes external data.

\begin{tabular}{l|ccc}
\hline Model & CLOTH & CLOTH-M & CLOTH-H \\
\hline Our model & $\mathbf{0 . 5 8 3}$ & $\mathbf{0 . 6 7 3}$ & $\mathbf{0 . 5 4 9}$ \\
w.o. rep. & 0.566 & 0.662 & 0.528 \\
w.o. hum. & 0.565 & 0.665 & 0.526 \\
w.o. rep. or hum. & 0.543 & 0.643 & 0.505 \\
\hline
\end{tabular}

Table 9: Ablation study on using the representativeness information (denoted as rep.) and the human-created data (denoted as hum.)

\subsection{Results}

We summarize performances of all models in Table 8. Our representativeness model outperforms all other models that do not use external data on CLOTH, CLOTH-H and CLOTH-M.

\subsection{Analysis}

In this section, we verify the effectiveness of the representativeness-based averaging by ablation studies. When we remove the representativeness information by setting $\alpha$ to infinity, the accuracy drops from 0.583 to 0.566 . When we further remove the human-created data so that only generated data is employed, the accuracy drops to 0.543 , similar to the performance of LM. The results further confirm that it is beneficial to incorporate human-created questions into training.

A sample of the predicted representativeness is shown in Figure $1^{7}$. Clearly, words that are too obvious have low scores, such as punctuation marks, simple words "a" and "the". In contrast, content words whose semantics are directly related to the context have a higher score, e.g., "same", "similar", "difference" have a high score when the difference between two objects is discussed and "secrets" has a high score since it is related to the subsequent sentence "does not want to share with others". Our prediction model achieves an F1 score of 36.5 on the test set, which is understandable since

\footnotetext{
${ }^{7}$ The script to generate the Figure is obtained at https://gist.github.com/ihsgnef/ f13c35cd46624c8f458a4d23589ac768
} 


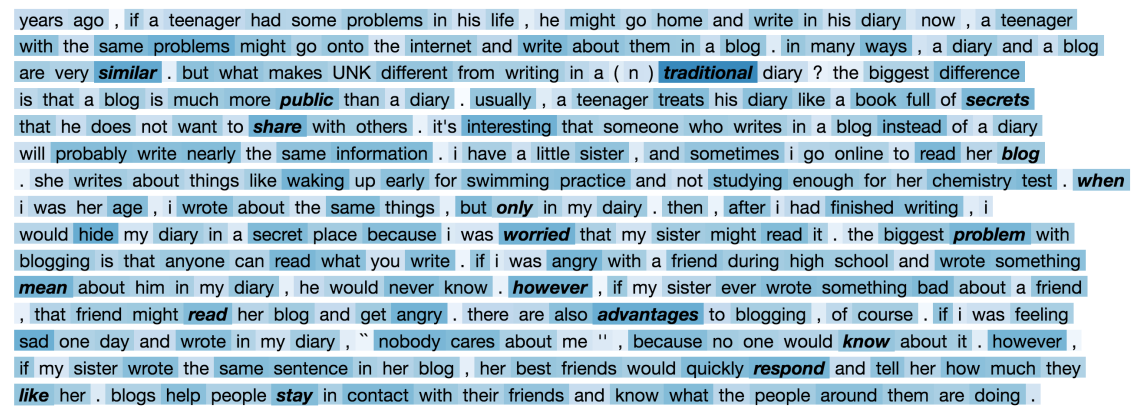

Figure 1: Representativeness prediction for each word. Lighter color means less representative. The words deleted by human as blanks are in bold text.

there are many plausible questions within a passage.

It has been shown that features such as morphology information and readability are beneficial in cloze test prediction (Skory and Eskenazi, 2010; Correia et al., 2012, 2010; Kurtasov, 2013). We leave investigating the advanced approaches of automatically designing cloze test to future work.

\section{Conclusion and Discussion}

In this paper, we propose a large-scale cloze test dataset CLOTH that is designed by teachers. With missing blanks and candidate options carefully created by teachers to test different aspects of language phenomena, CLOTH requires a deep language understanding and better captures the complexity of human language. We find that human outperforms 1B-LM by a significant margin. After detailed analysis, we find that the performance gap is due to the model's inability to understanding a long context. We also show that, compared to automatically-generated questions, human-created questions are more difficult and lead to a larger margin between human performance and the model's performance.

Despite the excellent performance of 1B-LM when compared with models trained only on CLOTH, it is still important to investigate and create more effective models and algorithms which provide complementary advantages to having a large amount of data. For rapid algorithm developments, we suggest training models only on the training set of CLOTH and comparing with models that do not utilize external data.

We hope our dataset provides a valuable testbed to the language modeling community and the machine comprehension community. In particular, the language modeling community can use
CLOTH to evaluate their models' abilities in modeling a long context. In addition, the machine comprehension community may also find CLOTH useful in evaluating machine's understanding of language phenomena including vocabulary, reasoning and grammar, which are key components of comprehending natural language.

In our future work, we would like to design algorithms to better model a long context, to utilize external knowledge, and to explore more effective semi-supervised learning approaches. Firstly, we would like to investigate efficient ways of utilizing external knowledge such as paraphrasing and semantic concepts like prior works (Dong et al., 2017; Dasigi et al., 2017). In comparison, training on a large external dataset is actually a time-consuming way of utilizing external knowledge. Secondly, to use the generated questions more effectively, the representative-based semisupervised approach might be improved by techniques studied in active learning and hard example mining (Settles, 2009; Shrivastava et al., 2016; Chang et al., 2017).

\section{Acknowledgement}

We thank Yulun Du, Kaiyu Shi and Zhilin Yang for insightful discussions and suggestions on the draft. We thank Shi Feng for the script to highlight representative words. This research was supported in part by DARPA grant FA8750-12-20342 funded under the DEFT program.

\section{References}

Dzmitry Bahdanau, Kyunghyun Cho, and Yoshua Bengio. 2014. Neural machine translation by jointly learning to align and translate. arXiv preprint arXiv:1409.0473. 
Haw-Shiuan Chang, Erik Learned-Miller, and Andrew McCallum. 2017. Active bias: Training more accurate neural networks by emphasizing high variance samples. In Advances in Neural Information Processing Systems, pages 1003-1013.

Ciprian Chelba, Tomas Mikolov, Mike Schuster, Qi Ge, Thorsten Brants, Phillipp Koehn, and Tony Robinson. 2013. One billion word benchmark for measuring progress in statistical lming. arXiv preprint arXiv:1312.3005.

Danqi Chen, Jason Bolton, and Christopher D Manning. 2016. A thorough examination of the $\mathrm{cnn} /$ daily mail reading comprehension task. arXiv preprint arXiv: 1606.02858 .

Peter Clark, Isaac Cowhey, Oren Etzioni, Tushar Khot, Ashish Sabharwal, Carissa Schoenick, and Oyvind Tafjord. 2018. Think you have solved question answering? try arc, the ai 2 reasoning challenge. arXiv preprint arXiv:1803.05457.

Rui Correia, Jorge Baptista, Maxine Eskenazi, and Nuno J Mamede. 2012. Automatic generation of cloze question stems. In PROPOR, pages 168-178. Springer.

Rui Correia, Jorge Baptista, Nuno Mamede, Isabel Trancoso, and Maxine Eskenazi. 2010. Automatic generation of cloze question distractors. In Proceedings of the Interspeech 2010 Satellite Workshop on Second Language Studies: Acquisition, Learning, Education and Technology, Waseda University, Tokyo, Japan.

Pradeep Dasigi, Waleed Ammar, Chris Dyer, and Eduard Hovy. 2017. Ontology-aware token embeddings for prepositional phrase attachment. arXiv preprint arXiv:1705.02925.

Bhuwan Dhingra, Hanxiao Liu, Zhilin Yang, William W Cohen, and Ruslan Salakhutdinov. 2016. Gated-attention readers for text comprehension. arXiv preprint arXiv:1606.01549.

Li Dong, Jonathan Mallinson, Siva Reddy, and Mirella Lapata. 2017. Learning to paraphrase for question answering. arXiv preprint arXiv:1708.06022.

Sandra S Fotos. 1991. The cloze test as an integrative measure of efl proficiency: A substitute for essays on college entrance examinations? Language Learning, 41(3):313-336.

Karl Moritz Hermann, Tomas Kocisky, Edward Grefenstette, Lasse Espeholt, Will Kay, Mustafa Suleyman, and Phil Blunsom. 2015. Teaching machines to read and comprehend. In NIPS.

Felix Hill, Antoine Bordes, Sumit Chopra, and Jason Weston. 2016. The goldilocks principle: Reading children's books with explicit memory representations. ICLR
Sepp Hochreiter and Jürgen Schmidhuber. 1997. Long short-term memory. Neural computation, 9(8):1735-1780.

Jon Jonz. 1991. Cloze item types and second language comprehension. Language testing, 8(1):1-22.

Mandar Joshi, Eunsol Choi, Daniel S Weld, and Luke Zettlemoyer. 2017. Triviaqa: A large scale distantly supervised challenge dataset for reading comprehension. $A C L$.

Rafal Jozefowicz, Oriol Vinyals, Mike Schuster, Noam Shazeer, and Yonghui Wu. 2016. Exploring the limits of lming. arXiv preprint arXiv:1602.02410.

Diederik Kingma and Jimmy Ba. 2014. Adam: A method for stochastic optimization. arXiv preprint arXiv: 1412.6980

Andrey Kurtasov. 2013. A system for generating cloze test items from russian-language text. In Proceedings of the Student Research Workshop associated with RANLP 2013, pages 107-112.

Guokun Lai, Qizhe Xie, Hanxiao Liu, Yiming Yang, and Eduard Hovy. 2017. Race: Large-scale reading comprehension dataset from examinations. EMNLP.

Tri Nguyen, Mir Rosenberg, Xia Song, Jianfeng Gao, Saurabh Tiwary, Rangan Majumder, and Li Deng. 2016. Ms marco: A human generated machine reading comprehension dataset. arXiv preprint arXiv:1611.09268.

Takeshi Onishi, Hai Wang, Mohit Bansal, Kevin Gimpel, and David McAllester. 2016. Who did what: A large-scale person-centered cloze dataset. arXiv preprint arXiv:1608.05457.

Denis Paperno, Germán Kruszewski, Angeliki Lazaridou, Quan Ngoc Pham, Raffaella Bernardi, Sandro Pezzelle, Marco Baroni, Gemma Boleda, and Raquel Fernández. 2016. The lambada dataset: Word prediction requiring a broad discourse context. arXiv preprint arXiv:1606.06031.

Adam Paszke, Sam Gross, Soumith Chintala, Gregory Chanan, Edward Yang, Zachary DeVito, Zeming Lin, Alban Desmaison, Luca Antiga, and Adam Lerer. 2017. Automatic differentiation in pytorch. In NIPS-W.

Anselmo Peñas, Yusuke Miyao, Álvaro Rodrigo, Eduard H Hovy, and Noriko Kando. 2014. Overview of clef qa entrance exams task 2014. In CLEF (Working Notes), pages 1194-1200.

Jeffrey Pennington, Richard Socher, and Christopher Manning. 2014. Glove: Global vectors for word representation. In EMNLP, pages 1532-1543.

Matthew E Peters, Mark Neumann, Mohit Iyyer, Matt Gardner, Christopher Clark, Kenton Lee, and Luke Zettlemoyer. 2018. Deep contextualized word representations. arXiv preprint arXiv:1802.05365. 
Pranav Rajpurkar, Jian Zhang, Konstantin Lopyrev, and Percy Liang. 2016. Squad: 100,000+ questions for machine comprehension of text. arXiv preprint arXiv:1606.05250.

Álvaro Rodrigo, Anselmo Peñas, Yusuke Miyao, Eduard H Hovy, and Noriko Kando. 2015. Overview of clef qa entrance exams task 2015. In CLEF (Working Notes).

J Sachs, P Tung, and RYH Lam. 1997. How to construct a cloze test: Lessons from testing measurement theory models. Perspectives.

Carissa Schoenick, Peter Clark, Oyvind Tafjord, Peter Turney, and Oren Etzioni. 2017. Moving beyond the turing test with the allen ai science challenge. Communications of the ACM, 60(9):60-64.

Minjoon Seo, Aniruddha Kembhavi, Ali Farhadi, and Hannaneh Hajishirzi. 2016. Bidirectional attention flow for machine comprehension. arXiv preprint arXiv:1611.01603.

Burr Settles. 2009. Active learning literature survey.

Hideyuki Shibuki, Kotaro Sakamoto, Yoshinobu Kano, Teruko Mitamura, Madoka Ishioroshi, Kelly Y Itakura, Di Wang, Tatsunori Mori, and Noriko Kando. 2014. Overview of the ntcir-11 qa-lab task. In NTCIR.

Abhinav Shrivastava, Abhinav Gupta, and Ross Girshick. 2016. Training region-based object detectors with online hard example mining. In Proceedings of the IEEE Conference on Computer Vision and Pattern Recognition, pages 761-769.

Adam Skory and Maxine Eskenazi. 2010. Predicting cloze task quality for vocabulary training. In Proceedings of the NAACL HLT 2010 Fifth Workshop on Innovative Use of NLP for Building Educational Applications, pages 49-56. Association for Computational Linguistics.

Wilson L Taylor. 1953. cloze procedure: a new tool for measuring readability. Journalism Bulletin, 30(4):415-433.

Annie Tremblay. 2011. Proficiency assessment standards in second language acquisition research. Studies in Second Language Acquisition, 33(3):339-372.

Adam Trischler, Tong Wang, Xingdi Yuan, Justin Harris, Alessandro Sordoni, Philip Bachman, and Kaheer Suleman. 2016. Newsqa: A machine comprehension dataset. arXiv preprint arXiv:1611.09830.

Wenhui Wang, Nan Yang, Furu Wei, Baobao Chang, and Ming Zhou. 2017. Gated self-matching networks for reading comprehension and question answering. In Proceedings of the 55th Annual Meeting of the Association for Computational Linguistics (Volume 1: Long Papers), volume 1, pages 189-198.
Yichong Xu, Jingjing Liu, Jianfeng Gao, Yelong Shen, and Xiaodong Liu. 2017. Towards human-level machine reading comprehension: Reasoning and inference with multiple strategies. arXiv preprint arXiv:1711.04964.

Yuhao Zhang, Victor Zhong, Danqi Chen, Gabor Angeli, and Christopher D Manning. 2017. Positionaware attention and supervised data improve slot filling. In Proceedings of the 2017 Conference on Empirical Methods in Natural Language Processing, pages $35-45$.

Yukun Zhu, Ryan Kiros, Rich Zemel, Ruslan Salakhutdinov, Raquel Urtasun, Antonio Torralba, and Sanja Fidler. 2015. Aligning books and movies: Towards story-like visual explanations by watching movies and reading books. In Proceedings of the IEEE international conference on computer vision, pages 19-27.

Geoffrey Zweig and Christopher JC Burges. 2011. The microsoft research sentence completion challenge. Technical report, Technical Report MSR-TR-2011129, Microsoft. 


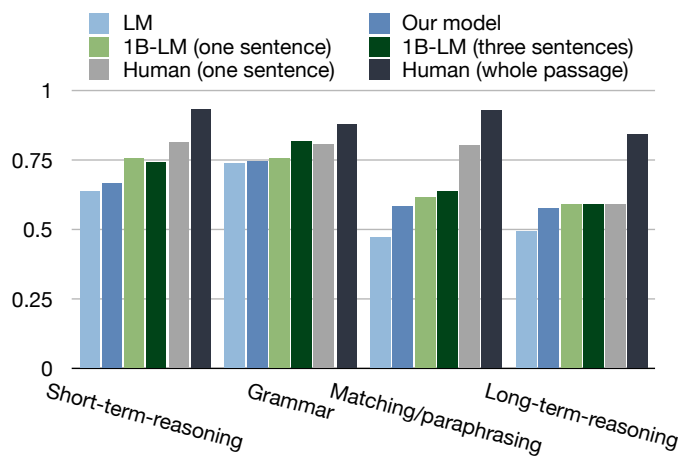

(a) Middle school group (CLOTH-M)

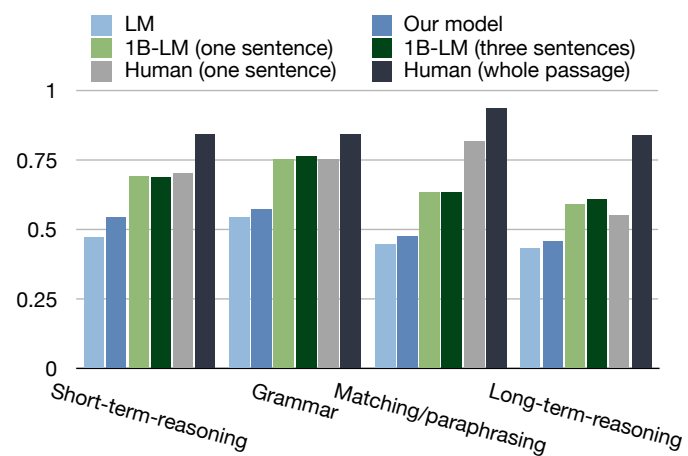

(b) High school group (CLOTH-H)

Figure 2: Model and human's performance on questions with different types. Our model will be introduced in Sec. 6.

\section{A Appendix}

\section{A.1 Question Type Labeling}

To label the questions, we provided the definition and an example for each question category to the Amazon Mechanical Turkers. To ensure quality, we limited the workers to master Turkers who are experienced and maintain a high acceptance rate. However, we did not restrict the backgrounds of the Turkers since master Turkers should have a reasonable amount of knowledge about English to conduct previous tasks. In addition, the vocabulary used in CLOTH are usually not difficult since they are constructed to test non-native speakers in middle school or high school. To get a concrete idea of the nature of question types, please refer to examples shown in Tab. 10.

\section{A.2 Type-specific Performance Analysis}

We can also further verify the strengths and weaknesses of the 1B-LM by studying the performance of models and human on different question categories. Note that the performance presented here may be subject to a high variance due to the limited number of samples in each category. From the comparison shown in Figure 2, we see that 1B-LM is indeed good at short-term questions. Specifically, when the human only has access to the context of one sentence, 1B-LM is close to human's performance on almost all categories. Further, comparing LM and 1B-LM, we find that training on the large corpus leads to improvements on all categories, showing that training on a large amount of data leads to a substantial improvement in learning complex language regularities.

\section{A.3 Implementation Details}

We implement our models using PyTorch (Paszke et al., 2017). We train our model on all questions in CLOTH and test it on CLOTH-M and CLOTH-H separately. For our final model, we use Adam (Kingma and $\mathrm{Ba}, 2014)$ with the learning rate of 0.001 . The hidden dimension is set to 650 and we initialize the word embedding by 300-dimensional Glove word vector (Pennington et al., 2014). The temperature $\alpha$ is set to 2 . We tried to increase the dimensionality of the model but do not observe performance improvement.

When we train the small LM on CLOTH, we largely follow the recommended hyperparameters in the Pytorch LM example ${ }^{8}$. Specifically, we employ a 2-layer LSTM with hidden dimension as 1024 . The input embedding and output weight matrix are tied. We set the dropout rate to 0.5 . The initial learning rate is set to 10 and divided by 4 whenever the PPL stops improving on the dev set.

We predict the answer for each blank independently for all of the models mentioned in this paper, since we do not observe significant performance improvements in our preliminary experiments when an auto-regressive approach is employed, i.e., when we fill all previous blanks with predicted answers. We hypothesize that, regardless of whether there exist inter-blank dependencies, since blanks are usually

\footnotetext{
${ }^{8}$ https://github.com/pytorch/examples/tree/master/word_language_model
} 
distributed far away from each other, LSTM is not able to capture such long dependencies. When testing language models, we use the longest text spans that do not contain blanks.

Passage: Nancy had just got a job as a secretary in a company. Monday was the first day she went to work, so she was very _1_ and arrived early. She _ 2 the door open and found nobody there. "I am the 3 - to arrive." She thought and came to her desk. She was surprised to find a bunch of _ 4 on it. They were fresh. She _5_ them and they were sweet. She looked around for a _6_ to put them in. "Somebody has sent me flowers the very first day!" she thought ${ }_{-} 7_{-}$. " But who could it be?" she began to _ $8_{-}$. The day passed quickly and Nancy did everything with _9_ interest. For the following days of the _ $10_{-}$, the first thing Nancy did was to change water for the followers and then set about her work.

Then came another Monday. _11_ she came near her desk she was overjoyed to see a(n) _12_bunch of flowers there. She quickly put them in the vase, ${ }_{-} 13_{-}$the old ones. The same thing happened again the next Monday. Nancy began to think of ways to find out the $1_{-} 4_{-}$. On Tuesday afternoon, she was sent to hand in a plan to the _15_. She waited for his directives at his secretary's _16_ . She happened to see on the desk a half-opened notebook, which _17_: "In order to keep the secretaries in high spirits, the company has decided that every Monday morning a bunch of fresh flowers should be put on each secretarys desk." Later, she was told that their general manager was a business management psychologist.

\begin{tabular}{|c|c|c|c|c|c|}
\hline \multicolumn{5}{|c|}{ Questions } & \multirow{2}{*}{$\begin{array}{l}\text { Question type } \\
\text { ort-term reasoning }\end{array}$} \\
\hline 1. & A. depressed & B. encouraged & C. excited & D. surprised & \\
\hline 2. & A. turned & B. pushed & C. knocked & D. forced & short-term reasoning \\
\hline 3. & A. last & B. second & C. third & D. first & long-term reasoning \\
\hline 4. & A. keys & B. grapes & C. flowers & D. bananas & matching \\
\hline 5. & A. smelled & B. ate & C. took & D. held & short-term reasoning \\
\hline 6. & A. vase & B. room & C. glass & ttle & long-te \\
\hline 7. & A. angrily & tly & gely & ppily & short- \\
\hline 8. & A. seek & nder & C. & ). & long-t \\
\hline 9. & A. low & B. 1 & C. great & neral & long-ter \\
\hline 10. & A. month & B. period & C. year & D. week & long-term reasoning \\
\hline 11. & A. Unless & B. When & C. Since & D. Before & grammar \\
\hline 12. & A. old & B. red & C. blue & D. $n$ & long-term reasoning \\
\hline 13. & vering & B. demanc & C. repla & bidding & long-terr \\
\hline 14. & A. sender & B. receiver & C. secretary & D. waiter & long-term reasoning \\
\hline 15. & A. assistant & B. colleague & C. employee & D. manager & matching \\
\hline 16 & A. notebook & B. desk & C. office & D. house & matching \\
\hline 17. & A. said & B. written & C. printed & D. signed & grammar \\
\hline
\end{tabular}

Table 10: An Amazon Turker's label for the sample passage 